\title{
Role of deformable image registration for delivered dose accumulation of adaptive external beam radiation therapy and brachytherapy in cervical
} cancer

\author{
Laura E van Heerden, MSc, Jorrit Visser, PhD, Kees Koedooder, PhD, Coen RN Rasch, MD PhD, Bradley R Pieters, MD PhD, \\ Arjan Bel, PhD \\ Department of Radiation Oncology. Amsterdam UMC, University of Amsterdam, Amsterdam, The Netherlands
}

\begin{abstract}
Purpose: Deformable image registration (DIR) can be used to accumulate the absorbed dose distribution of daily image-guided adaptive external beam radiation treatment (EBRT) and brachytherapy (BT). Since dose-volume parameter addition assumes a uniform delivered EBRT dose around the planned BT boost, the added value of DIR over direct addition was investigated for dose accumulation in bladder and rectum.

Material and methods: For 10 patients (EBRT 46/46.2 $\mathrm{Gy}_{\mathrm{EQD} 2}$, EBRT + BT: $\mathrm{D}_{90}$ 85-90 Gy $\mathrm{E}_{\mathrm{EQD} 2}$, in equivalent dose in 2 Gy fractions), the actually delivered dose from adaptive volumetric-modulated arc therapy (VMAT)/intensity-modulated radiotherapy (IMRT) EBRT was calculated using the daily anatomy from the cone-beam computed tomography (CBCT) scans acquired prior to irradiation. The CBCT of the first EBRT fraction and the BT planning MRI were registered using DIR. The cumulative dose to the $2 \mathrm{~cm}^{3}$ with the highest dose $\left(\mathrm{D}_{2 \mathrm{~cm}}\right)$ from EBRT and BT to the bladder and rectum was calculated and compared to direct addition assuming a uniform EBRT dose (UD).

Results: Differences (DIR-UD) in the total EBRT + BT dose ranged between -0.2-3.9 Gy $\mathrm{EQD}_{\mathrm{EQD}}$ (bladder) and -1.03.7 Gy $\mathrm{EQD2}_{\mathrm{E} 2}$ (rectum). The total EBRT + BT dose calculated with DIR was at most $104 \%$ of the dose calculated with the UD method.

Conclusions: Differences between UD and DIR were small $\left(<3.9 \mathrm{~Gy}_{\mathrm{EQD} 2}\right)$. The dose delivered with adaptive VMAT/IMRT EBRT to bladder and rectum near the planned BT boost can be considered uniform for the evaluation of bladder/rectum $\mathrm{D}_{2 \mathrm{~cm} 3}$.

J Contemp Brachytherapy 2018; 10, 6: 542-550 DOI: https://doi.org/10.5114/jcb.2018.79840
\end{abstract}

Key words: brachytherapy, cervical cancer, dose accumulation, deformable image registration.

\section{Purpose}

Locally-advanced cervical cancer is treated with concurrent chemotherapy and radiotherapy. The standard of care for radiotherapy is external beam radiation treatment (EBRT) and a brachytherapy (BT) boost to the tumor area using an intracavitary/interstitial applicator. The recommended EBRT dose to the target is 45-50 Gy in 1.8-2.0 Gy/fraction [1], with a BT boost up to a total dose of at least $90 \mathrm{~Gy}_{\mathrm{EOD} 2}$, expressed as equivalent dose in 2 Gy fractions $\left(\mathrm{EQD}_{2}\right)$ to at least $90 \%$ of the clinical target volume at high-risk (CTV-HR). To avoid toxicity, the cumulative dose to the $2 \mathrm{~cm}^{3}$ with the highest dose $\left(D_{2 \mathrm{~cm}}\right)$ from EBRT and BT to the bladder and rectum should not exceed $90 \mathrm{~Gy}_{\mathrm{EQD} 2}$ and $75 \mathrm{~Gy}_{\mathrm{EQD} 2}$, respectively $[2,3]$.

To evaluate the cumulative bladder and rectum $\mathrm{D}_{2 \mathrm{~cm} 3}$ of EBRT and BT, the International Commission on Radi- ation Units and Measurements (ICRU) recommends that the EBRT dose to the organs at risk should be considered uniform and equal to the prescription dose, meaning that the EBRT prescription dose and the BT dose-volume histogram (DVH) parameters can simply be added [4]. However, intensity-modulated radiotherapy (IMRT) and volumetric-modulated arc therapy (VMAT) as well as adaptive EBRT strategies such as plan-of-the-day strategies [5], are being increasingly used to create highly conformal dose distributions (Figure 1A). Possibly, the delivered dose from EBRT to organ at risk (OAR) is non-uniform near the location of the planned BT boost, causing the estimated cumulative $\mathrm{D}_{2 \mathrm{~cm} 3}$ to be inaccurate at the time of brachytherapy planning. This might lead to errors in establishing the dose-response relationship. It should therefore be investigated whether it is preferable
Address for correspondence: Laura E van Heerden, MSc, Department of Radiation Oncology, Amsterdam UMC, University of Amsterdam, AMC location, Meibergdreef 9, 1105 AZ Amsterdam, The Netherlands, phone: +31 24351678, e-mail: 1.e.vanheerden@amc.uva.nl
Received: 01.09 .2018

Accepted: 03.11.2018

Published: 28.12.2018 

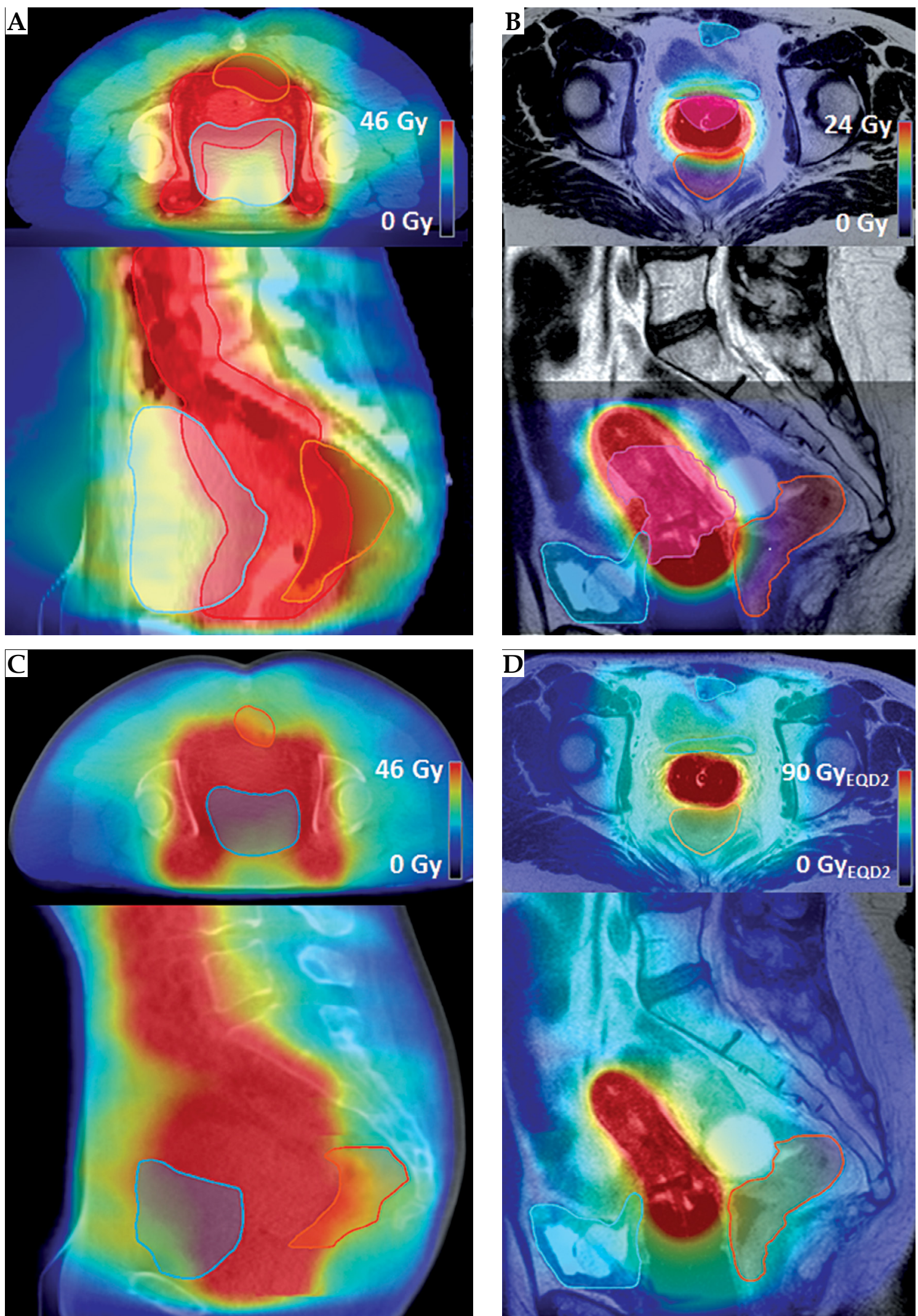

Fig. 1. A, B) Axial (upper panel) and sagittal (lower panel) view of a patient CT/MRI with a color wash of the planned VMAT/ BT dose. The VMAT dose is not uniform in the proximity of the bladder/rectum walls (blue/orange) closest to the target (red/ pink). C) СBCT of the first fraction with the total delivered dose from EBRT accumulated using DIR for the bladder as described in this paper. D) MRI with the accumulated dose from EBRT and BT, which is calculated by summing the delivered VMAT and planned BT dose distribution. Color version available 
to sum the 3D dose distributions instead of using the uniform dose (UD) method.

When accumulating the total dose, deformable image registration (DIR) can be used to account for deformation due to differences in bladder and rectum filling and/or the presence of air as well as the effect of the applicator on the position of the bladder and rectum. In earlier studies $[6,7,8,9,10]$, the added value of DIR for the calculation of cumulative bladder and rectum $\mathrm{D}_{2 \mathrm{~cm} 3}$ was investigated and small differences $(<5 \%)$ with the UD method were found. However, in these previous studies, the planning images of BT and EBRT were registered and subsequently the planned EBRT dose was summed with the planned BT dose. At the time of BT planning, the delivered EBRT dose may deviate from planned dose due to daily positioning variability and daily variation in organ filling. Moreover, for clinical patients treated with a plan-of-theday strategy, the delivered EBRT dose near the location of the planned BT boost has not yet been investigated. A plan-of-the-day strategy in combination with smaller margins may lead to a non-uniform delivered EBRT dose to the BT high-dose volumes.

The impact of EBRT dose non-uniformity in the planned BT high-dose volumes has never been investigated before, while taking into account the daily interfraction motion during EBRT. In the present study, we calculate the delivered EBRT dose for each fraction using the daily anatomy from the cone-beam computed tomography $(\mathrm{CBCT})$ scan acquired prior to irradiation, and then sum the accumulated delivered EBRT dose to the planned BT 3D dose distribution.

The aim of the present study is therefore to evaluate an adaptive EBRT planning procedure, if it is necessary to account for EBRT dose non-uniformity with deformable image registration when evaluating the cumulative EBRT and BT dose in bladder and rectum.

\section{Material and methods}

\section{Patients, dose scheme, and imaging}

In this study, ten patients treated for locally-advanced cervical carcinoma (FIGO stages IIA-IVA) were investigated. The patients were treated with EBRT, receiving $46 \mathrm{~Gy}$ in daily fractions of $2 \mathrm{~Gy}$ (8 patients) or $46.2 \mathrm{~Gy}$ in $1.65 \mathrm{~Gy}$ fractions, with a para-aortal boost up to $56 \mathrm{~Gy}$ in $2 \mathrm{~Gy}$ fractions (2 patients), and a BT boost. For patients treated with 46 Gy in 2 Gy fractions, five received an additional simultaneous-integrated boost (SIB) of 0.4 Gy per fraction to affected lymph nodes. These lymph nodes were located in the region between the aortic bifurcation above the internal/external iliac, and the dose to these nodes will not contribute to the BT boost region. All patients were treated according to a plan-of-the-day strategy [11]. Besides two planning CTs (i.e., full and empty bladder), all patients received $\mathrm{CBCT}$ imaging (Synergy platform, Elekta AB, Stockholm, Sweden) before irradiation. For both planning $\mathrm{CTs}$, the corresponding primary CTVs (pCTVs), consisting of the gross tumor volume, cervix, corpus-uterus, and upper part of the vagina, were registered using a structure-based DIR algorithm [12]. With the resulting deformation vector field, 1-3 patient-specific primary internal target volumes (pITVs) were generated. For each pITV, a primary planning target volume (PTV) was generated by enlarging the part of the pITV including the corpus-uterus with an $8 \mathrm{~mm}$ margin and the part of the pITV, including the cervix and vagina with a margin of $8 \mathrm{~mm}, 8 \mathrm{~mm}$, and $13 \mathrm{~mm}$ in left-right, superior-inferior, and anterior-posterior direction, respectively [11].

A patient-specific plan library was defined by generating 1-3 plans corresponding to the different target volumes. Each treatment day the library plan best fitting the anatomy as observed on the pre-fraction CBCT image was selected.

EBRT was planned with volumetric modulated arc therapy (VMAT) for nine patients, and with intensity modulated radiation therapy (IMRT) for one patient. For all EBRT plans (46 Gy $\mathrm{EQD}_{2}, 46 \mathrm{~Gy}_{\mathrm{EQD} 2}+\mathrm{SIB}$ or 46.2 $\mathrm{Gy}_{\mathrm{EQD} 2}$ ), the same bladder and rectum dose constraints were applied. For the target, $\geq 99 \%$ of the PTV should receive $\geq 95 \%$ of the prescription dose, with a conformity index $\left(\mathrm{V}_{95} / \mathrm{PTV}\right.$ volume $)<1.35$. A hotspot criterion was used for bladder and rectum $\left(D_{1 \mathrm{~cm} 3}<103 \%\right.$ of the prescription dose, i.e., $46 \mathrm{~Gy} / 46.2 \mathrm{~Gy})$. The dose fall-off region from 45-22.5 Gy was aimed to be $<1.2 \mathrm{~cm}$.

For brachytherapy, a dose of 24 Gy in pulse doses of 1 Gy every hour was delivered to the high-risk CTV $\left(\mathrm{CTV}_{\mathrm{HR}}\right)$ using the Utrecht CT/MR compatible applicator (Elekta, Veenendaal, The Netherlands), with vaginal ovoids and interstitial needles where needed for lateral coverage. Prior to the BT delivery, $\mathrm{T}_{2}$-weighted Turbo Spin Echo MRI (in-plane resolution $0.5 \times 0.5 \mathrm{~mm}^{2}$, slice thickness $3.3 \mathrm{~mm}$ ) was acquired on an Ingenia 3T MRI scanner (Philips Healthcare, Best, The Netherlands) [13]. Brachytherapy planning was performed using Oncentra Brachy 4.5 (Elekta, Veenendaal, The Netherlands), using a library for applicator reconstruction. The plan was manually optimized aiming at a cumulative $\mathrm{D}_{90}$ (minimal dose received by $90 \%$ of the volume) of $90-95 \mathrm{~Gy}_{\mathrm{EQD} 2}$ from EBRT and BT on the CTV $\mathrm{HR}_{\mathrm{HR}}$. To spare the bladder and rectum, the planned cumulative $\mathrm{D}_{2 \mathrm{~cm} 3}$ from EBRT and BT should not exceed $90 \mathrm{~Gy}_{\mathrm{EQD} 2}$ and $75 \mathrm{~Gy}_{\mathrm{EQD} 2}$, respectively. The radiotherapy techniques are described in Table 1 , and typical planned EBRT and BT dose distributions are shown in Figure 1 (A and B).

On the computed tomography (CT), CBCT and magnetic resonance imaging (MRI) scans, the bladder was delineated, and the rectum was delineated from the rectosigmoid junction to the level of the anal sphincter. For EBRT, patients were instructed to have a full bladder daily, while a catheter was inserted for BT to guarantee a minimum bladder filling. No rectal management was applied for BT dose delivery.

\section{Study-specific daily dose calculation of external beam radiotherapy}

For all 10 patients, CBCTs of all fractions were available, in total 240 images. Accurate CBCT-based dose calculation is difficult with the Elekta Synergy CBCT system used in this study because the Hounsfield Units (HU) numbers are not accurate. To enable daily dose 
Table 1. Radiotherapy techniques for EBRT and PDR BT

\begin{tabular}{|c|c|c|}
\hline & External beam radiation therapy & Brachytherapy \\
\hline Treatment type & VMAT/IMRT & PDR \\
\hline Treatment planning system & Oncentra $4.3^{\star}$ & Oncentra Brachy $4.5^{*}$ \\
\hline Dose calculation algorithm & Collapsed cone & Based on the TG-43 formalism \\
\hline Imaging & CT images & $\mathrm{T}_{2}$-weighted Turbo Spin Echo MRI \\
\hline Scanner specification & LightSpeed RT16 (GE, WI, USA) & $\begin{array}{c}\text { Ingenia 3T (Philips Healthcare, Best, } \\
\text { The Netherlands) }\end{array}$ \\
\hline Imaging resolution $\left(\mathrm{mm}^{3}\right)$ & $1.2 \times 1.2 \times 3$ & $0.7 \times 0.7 \times 3.3$ \\
\hline Dose calculation grid size $\left(\mathrm{mm}^{3}\right)$ & $3 \times 3 \times 3$ & $1 \times 1 \times 1$ \\
\hline Planning aims & $\begin{array}{c}\text { PTV } \mathrm{V}_{95} \geq 99 \% \\
\text { Bladder } \mathrm{V}_{45 \mathrm{~Gy}}<40 \% \\
\text { Rectum } \mathrm{V}_{40 \mathrm{~Gy}}<70 \% \\
\text { Bladder + rectum } \mathrm{D}_{1 \mathrm{~cm} 3}<103 \% \\
\text { of the prescription dose }\end{array}$ & $\begin{array}{c}\mathrm{CTV}_{\mathrm{HR}} \mathrm{D}_{90} 85-90 \mathrm{~Gy}_{\mathrm{EQD2}} \\
\text { Bladder/rectum } \mathrm{D}_{2 \mathrm{~cm} 3}<80 / 65 \mathrm{~Gy}_{\mathrm{EQD2}}\end{array}$ \\
\hline
\end{tabular}

*Elekta $A B$, Stockholm, Sweden

PTV - planning target volume; $C T V_{H R}$ - high-risk clinical target volume; $D_{90}$ - minimal dose received by $90 \%$ of the volume; $V_{95}$-the percentage of the volume receiving $\geq 95 \%$ of the prescription dose; $V_{45 G y} / V_{40 G y}$ - the percentage of the volume receiving $\geq 45 / 40 \mathrm{~Gy} ; D_{2 \mathrm{~cm}_{3}} / D_{1 \mathrm{~cm}_{3}}-$ minimal dose to $2 \mathrm{~cm}^{3} / 1 \mathrm{~cm}^{3}$ with the highest dose; EQD2 - equivalent dose at $2 \mathrm{~Gy}$
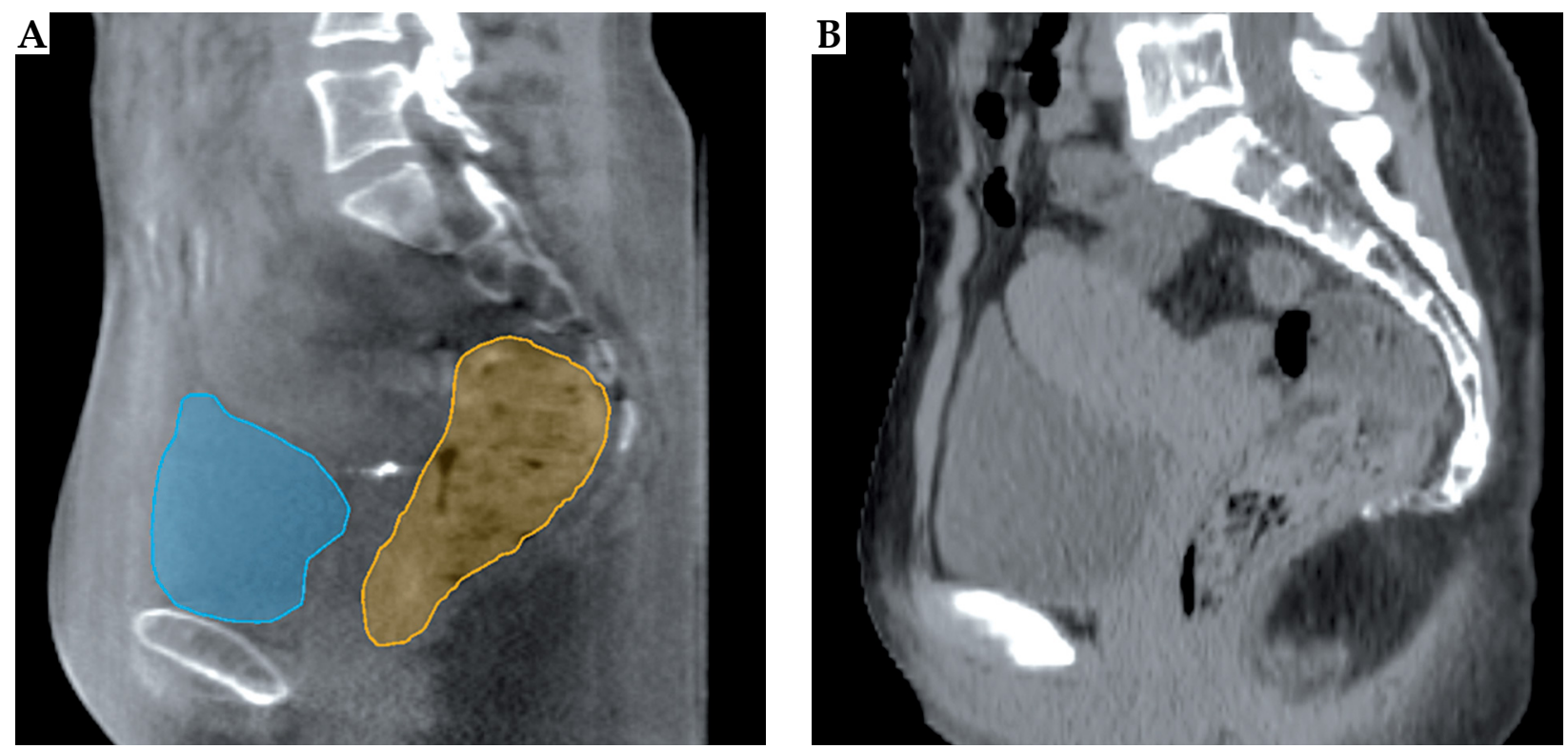

Fig. 2. A) Sagittal view of a patient $C B C T$ with the bladder/rectum delineations (blue/orange). B) The planning CT with accurate $\mathrm{HU}$ numbers deformed to the $\mathrm{CBCT}$ frame of reference. Color version available online

distribution calculation for all images, CT HU numbers were mapped to $\mathrm{CBCT}$ images by registering the planning CT to CBCT images using DIR (Figure 2) [14]. For each pre-fraction $\mathrm{CBCT}$ image, the planning $\mathrm{CT}$ with accurate $\mathrm{HU}$ numbers was deformed to represent CBCT images using a B-spline deformable image registration based on intensity values (VelocityAI, version 3.1.0/3.2.0, Varian Medical Systems, Inc., Palo Alto, CA). Of the two available planning CT's (i.e., full and empty bladder), we selected the CT with the closest bladder volume to the daily anatomy of the CBCT for this step. Prior to the deformable image registration, a rigid registration was performed to match the bony anatomy. The deformable match was visually assessed to ensure that the body contours and the soft tissue matched sufficiently. The quality of CT-to-CBCT deformable registration in the pelvic area using the VelocityAI software was investigated previously and DIR results were reported to be accurate for dose calculation $[15,16]$.

Each daily selected plan was used to calculate the corresponding daily dose distribution.

\section{Deformable image registration for dose accumulation}

To accumulate the delivered EBRT dose and EBRT + BT dose, the structure-guided DIR available in VelocityAI 
was used. This is a hybrid version of the B-spline DIR, where a higher weight is assigned to voxels within the structure. Our method is similar to earlier described DIR strategies for dose accumulation $[17,18]$. To obtain DIR of sufficient quality, all delineated EBRT and BT images were converted into binary images before DIR for bladder and rectum separately. Two registrations for each patient were created for bladder and rectum separately to improve the quality of each registration in the proximity of the evaluated organ.

First, all CBCT images were registered to the CBCT of the first fraction (CBCT1) using deformable image registration. A mapping was established to propagate delineated structures on each subsequent CBCT to CBCT1 scan. Next, the CBCT1 image was deformably registered to the BT planning MRI to obtain a mapping of delineated structures on CBCT1 to the BT MRI.

\section{Dose accumulation}

For both organ-specific matches, the deformation vector fields (DVFs) obtained with the CBCT-to-CBCT DIR were used to map all EBRT fraction doses to the CBCT1 frame of reference. This is the delivered dose distribution from all EBRT fractions (Figure 1C). The CBCT1-to-MRI DVF was used to map the delivered EBRT dose distribution and organ contours to the frame of reference of the planned BT dose distribution. Next, the EBRT and BT doses were converted to $\mathrm{EQD}_{2}$ on a voxel-by-voxel level (Matlab R2014b, Mathworks Inc., MA) using LQ-modelbased equations with an $\alpha / \beta$ value of $3 \mathrm{~Gy}$ for late OAR toxicity and a 1.5 hour repair half-time $[19,20]$. Finally, the EBRT and BT doses were summed to create the accumulated dose (Figure 1D). Figure 3 shows a schematic overview of the workflow.

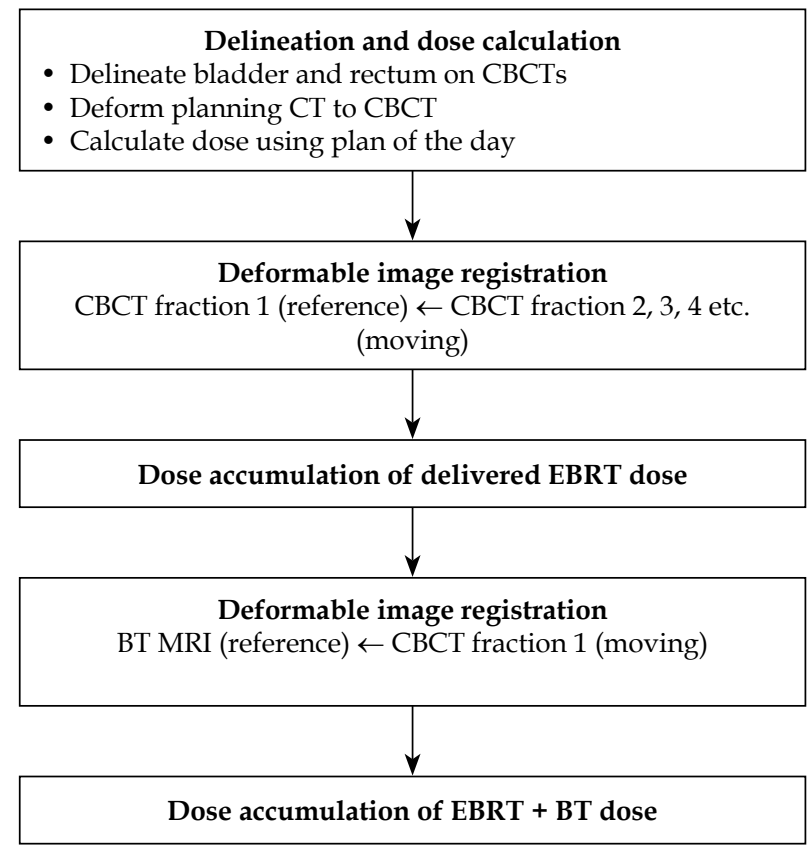

Fig. 3. Schematic overview of the workflow for accumulating the EBRT + BT dose

\section{Dosimetric data analysis}

Bladder and rectum $\mathrm{D}_{2 \mathrm{~cm} 3}$ were acquired using VelocityAI. For the DIR method, $\mathrm{D}_{2 \mathrm{~cm} 3}$ was calculated from the accumulated dose distributions for both rectum and bladder. For the UD method, the cumulative $\mathrm{D}_{2 \mathrm{~cm} 3}$ was calculated by adding the planned dose of $46 \mathrm{~Gy}_{\mathrm{EQD} 2} / 46.2$ $\mathrm{Gy}_{\mathrm{EQD} 2}$ to the $\mathrm{D}_{2 \mathrm{~cm} 3}$ from BT. For both methods, the mean and range of bladder and rectum $\mathrm{D}_{2 \mathrm{~cm} 3}$ were calculated for all patients as well as the difference in $\mathrm{D}_{2 \mathrm{~cm} 3}$ (DIR-UD).

\section{DIR accuracy}

The dice similarity coefficient (DSC) [21] was used to compare propagated and reference contours. DSC quantifies the spatial overlap of the matched bladder and rectum. Additionally, the surface distance error (SDE), i.e., the Euclidean distance between the reference and propagated contours, was determined for each bladder/rectum match $[22,23]$. Over all bladder/rectum matches, the mean DSC and range over all patients were calculated. The $25^{\text {th }}, 50^{\text {th }}, 75^{\text {th }}$, and $100^{\text {th }}$ percentile of the mean SDEs were calculated for all bladders/rectums.

\section{Results}

\section{Cumulative DVH parameters}

The $\mathrm{D}_{2 \mathrm{~cm} 3}$ mean and range for bladder and rectum are shown in Table 2 . The difference range (DIR-UD) was $-0.2-3.7 \%\left(-0.2-3.9 \mathrm{~Gy}_{\mathrm{EQD} 2}\right)$ for bladder and $-1.9-3.7 \%$ $\left(-1.0-3.7 \mathrm{~Gy}_{\mathrm{EQD} 2}\right)$ for rectum, meaning that the dose to the OAR calculated with DIR was at most $104 \%$ of the dose calculated with the UD method. Individual patient results can be found in Figure 4.

\section{DIR accuracy}

For every patient, the mean DSC over all bladder/rectum matches was calculated. For the bladder, the mean DSC was 0.96, ranging between 0.95-0.97 over all patients. The mean rectum DSC was 0.92 (range, 0.89-0.94).

For the bladder, the mean SDE over all registrations was $0.7 \mathrm{~mm}$, ranging between $0.5-0.9 \mathrm{~mm}$. For the rectum,

Table 2. The mean and the range over all patients of the cumulative $D_{2 \mathrm{~cm}^{3}}$ calculated with the DIR method and the UD method, and the difference $\Delta D_{2 \mathrm{~cm}^{3}}$ (DIR-UD), in bladder and rectum

\begin{tabular}{lcc} 
Bladder & \multicolumn{1}{c}{$\begin{array}{l}\text { DIR } \\
\text { Method }\end{array}$} & $\begin{array}{c}\text { Uniform dose } \\
\text { Mean (range) }\end{array}$ \\
\hline $\mathrm{D}_{2 \mathrm{~cm} 3}\left(\mathrm{~Gy}_{\mathrm{EQD2} 2}\right)$ & $75.6(57.4-106.9)$ & $74.5(56.6-103.2)$ \\
\hline$\Delta \mathrm{D}_{2 \mathrm{~cm} 3}\left(\mathrm{~Gy} \mathrm{EQD2}_{2}\right)$ & - & $-1.1(-3.7-0.2)$ \\
\hline Rectum & & \\
\hline Method & DIR & Uniform dose \\
& Mean (range) & Mean (range) \\
\hline $\mathrm{D}_{2 \mathrm{~cm} 3}\left(\mathrm{~Gy}_{\mathrm{EQD2} 2}\right)$ & $63.2(50.6-74.7)$ & $63.3(49.7-77.5)$ \\
\hline$\Delta \mathrm{D}_{2 \mathrm{~cm} 3}\left(\mathrm{~Gy} \mathrm{EQD2}_{2}\right)$ & - & $0.0(-2.8-1.0)$
\end{tabular}

$D_{2 \mathrm{~cm} 3} / D_{1 \mathrm{~cm} 3}$ - minimal dose to $2 \mathrm{~cm}^{3} / 1 \mathrm{~cm}^{3}$ with the highest dose; $E Q D 2$ - equivalent dose at 2 Gy 


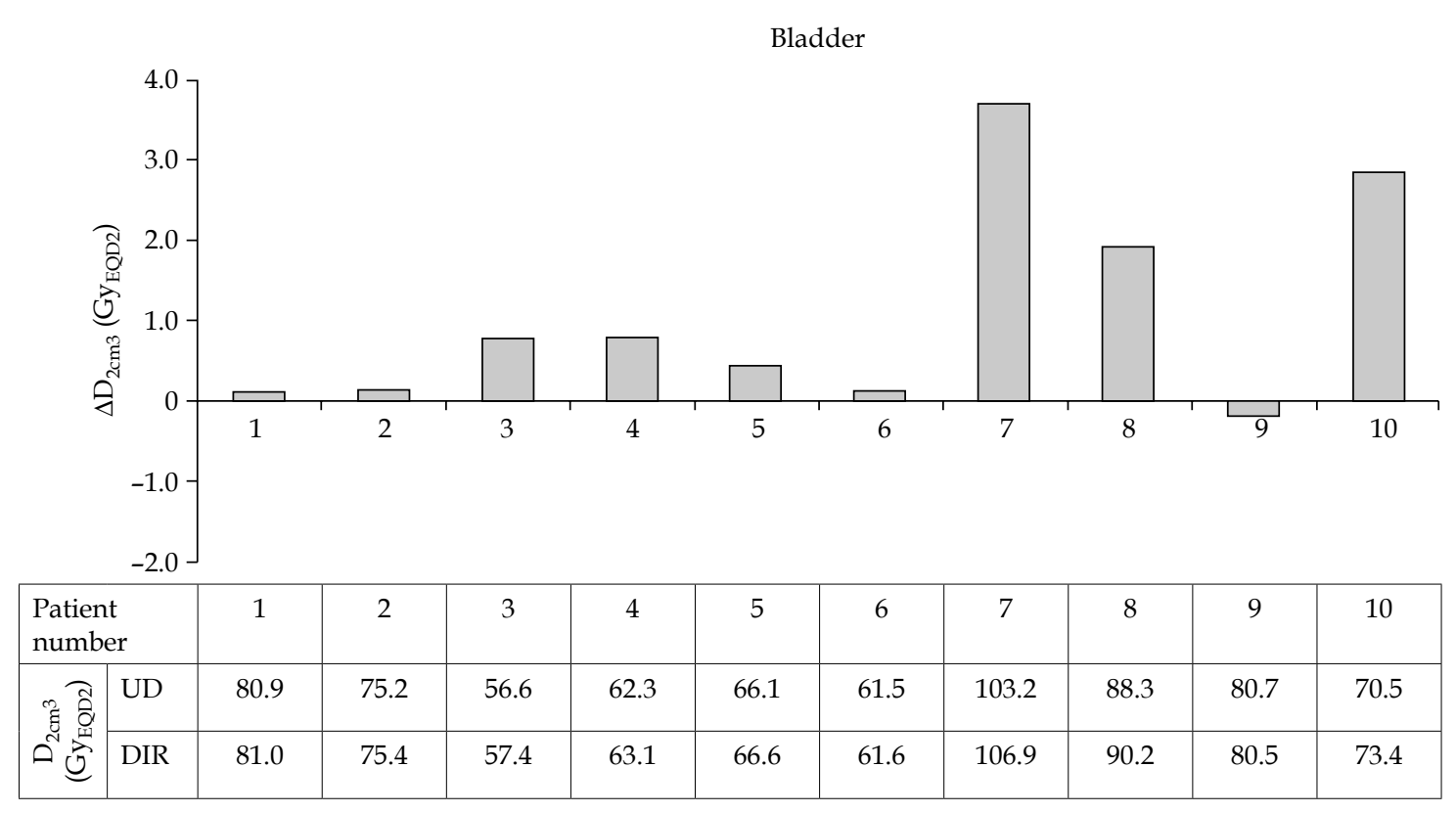

$\square$ DIR-UD

Rectum

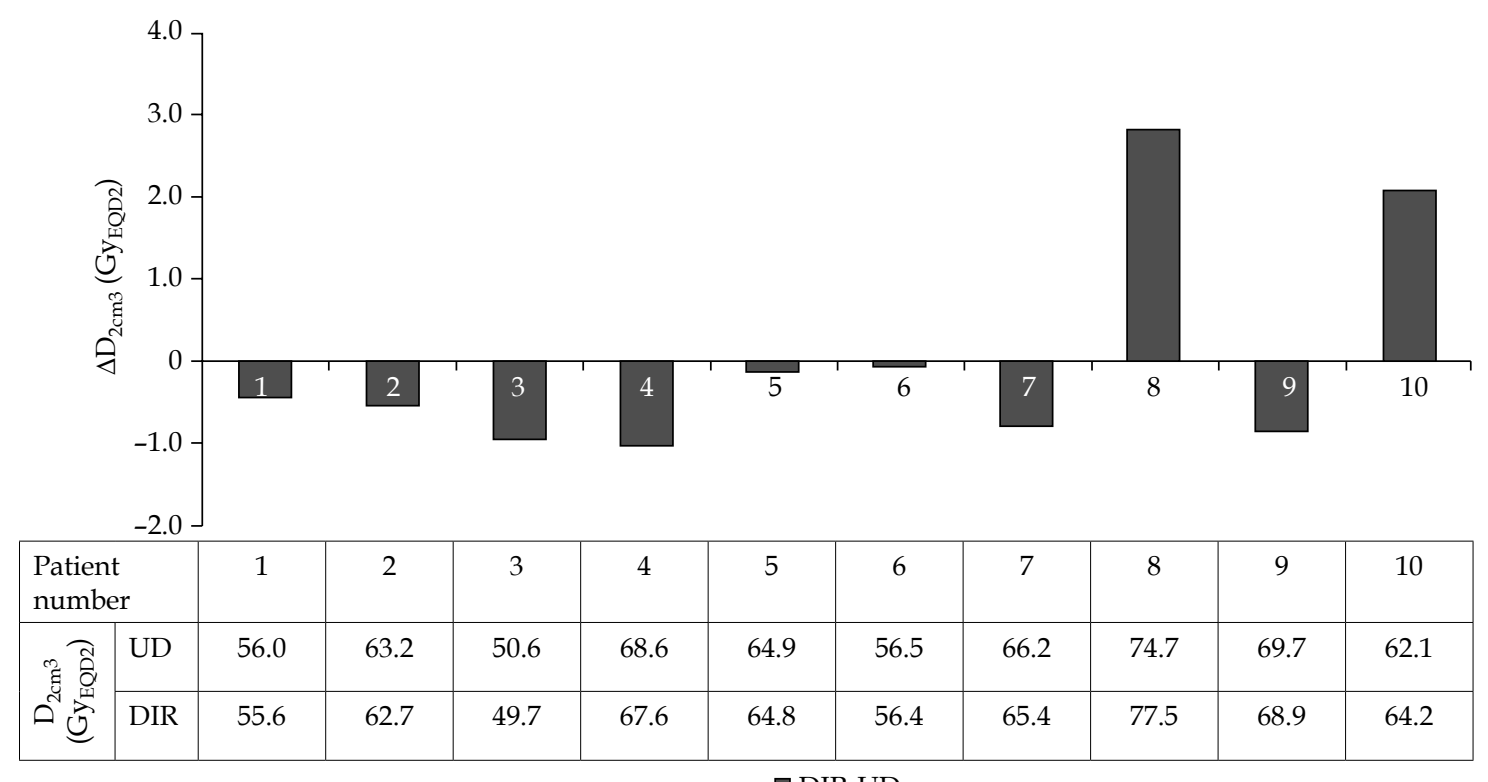

$\square$ DIR-UD

Fig. 4. The cumulative $\mathrm{D}_{2 \mathrm{~cm} 3}$ calculated with the DIR and UD method for bladder and rectum. For visualization, the difference in $\mathrm{D}_{2 \mathrm{~cm} 3}$ of the DIR method with the UD method $\left(\Delta \mathrm{D}_{2 \mathrm{~cm} 3}\right)$ is plotted

the mean SDE was $0.9 \mathrm{~mm}$ (range, 0.4-1.9 mm). Individual patient results are shown in Figure 5. For the CBCT1to-MRI registration, which was used to map the delivered EBRT dose distribution to the frame of reference of the planned BT dose distribution, the DSC ranged over all patients between 0.94-0.98 for bladder and for rectum between 0.89-0.92. The mean SDE varied for bladder between $0.3-0.7 \mathrm{~mm}$ and for the rectum between $1.0-1.9 \mathrm{~mm}$.

\section{Discussion}

In the present study, we investigated if it is necessary to account for non-uniformity of the dose from adaptive
EBRT with deformable image registration when evaluating the cumulative bladder and rectum $\mathrm{D}_{2 \mathrm{~cm} 3}$ from EBRT and BT at the time of BT planning. To our knowledge, this is the first study to estimate the delivered dose from EBRT and to compare the $\mathrm{D}_{2 \mathrm{~cm} 3}$ from the accumulated EBRT and BT 3D dose distribution to the UD method for patients treated with a plan-of-the-day strategy. For the total EBRT + BT dose, small differences were found between UD and DIR $\left(\Delta \mathrm{D}_{2 \mathrm{~cm} 3}<3.9 \mathrm{~Gy}_{\mathrm{EQD} 2}\right)$.

There are many uncertainties related to brachytherapy planning for cervical cancer [24,25]. Delineation uncertainty, inter- and intrafraction motion, and applicator 

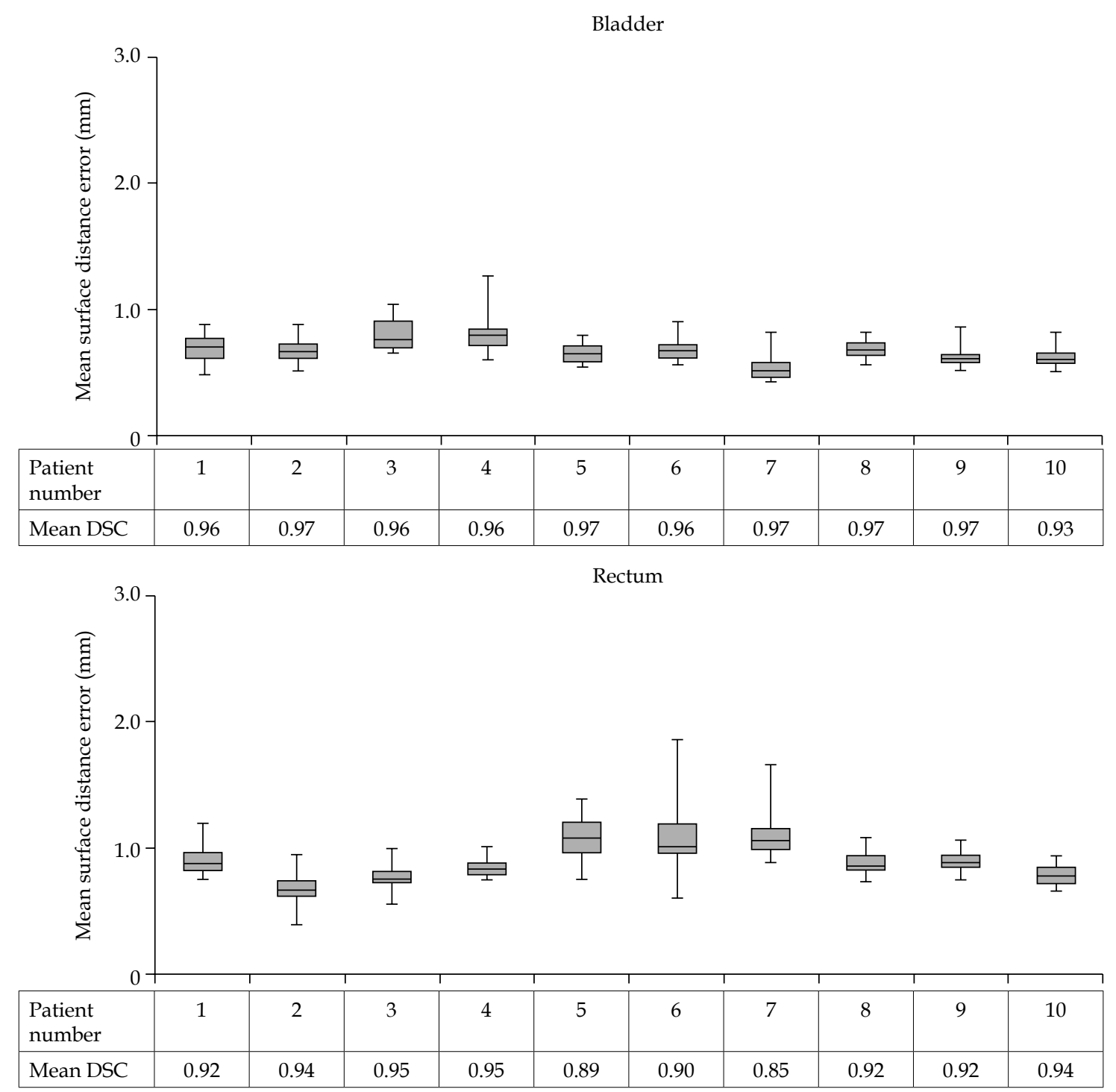

Fig. 5. Boxplot of the mean surface distance errors with the $25^{\text {th }}, 50^{\text {th }}, 75^{\text {th }}$, and $100^{\text {th }}$ percentile over all fractions, for all patients in bladder and rectum. The table shows the mean dice similarity coefficient (DSC) over all fractions

reconstruction uncertainty because of e.g., image distortions, are major contributors to the total uncertainty for the estimation of the total radiotherapy dose [26,27]. Calculation of the accumulated dose to bladder and rectum with the ICRU formalism [20] may lead to additional uncertainty, since it assumes that the delivered EBRT dose is uniform. In this paper, we accumulated the dose from EBRT and BT using DIR, while taking into account interfraction motion between EBRT fractions. Since we found small differences between the DIR and UD method, this study shows that using the ICRU formalism leads to small additional uncertainty for the estimated total EBRT and BT dose.

In previous studies, the dose effect relationship for late side effects was determined for bladder and rectum using the UD method [28,29]. Regardless of whether UD or DIR was used to calculate $\mathrm{D}_{2 \mathrm{~cm} 3}$, in our study, the risk remained within $5-10 \%$ for nine out of ten patients. For one patient, the cumulative bladder dose exceeded the dose limit of $90 \mathrm{~Gy}_{\mathrm{EQD} 2}$ and was as high as $103 \mathrm{~Gy}_{\mathrm{EQD} 2}$, since the bladder wall was part of the target. For another patient, the rectum dose was relatively high, with a value of $74.6 \mathrm{~Gy}_{\mathrm{EQD} 2}$. Still, the risk of side effects remained within 11-14\% with DIR or UD. Based on these results, we conclude that DIR provides no added value over the UD method for the evaluation of bladder/rectum $D_{2 \mathrm{~cm} 3}$ in order to predict organ toxicity.

For dose warping purposes, it is desirable to obtain high voxel-to-voxel correspondence after DIR. With our current methods, we did not directly investigate the voxel-to-voxel correspondence, and the DIR method used for dose accumulation was not validated using ground truth data. After DIR, we found a high mean DSC (bladder: 0.96, rectum: 0.92) and small mean SDE (bladder: $0.7 \mathrm{~mm}$, rectum: $0.9 \mathrm{~mm}$ ) for both bladder and rectum. For the evaluation of $\mathrm{D}_{2 \mathrm{~cm} 3}$, it is relevant to know whether mis- 
matches are located near the BT boost region. Visual assessment showed that for all registrations, the contours were overlapping near the BT boost. The mean SDE distribution showed that for all registrations, the distance between points on the matched contours was small after DIR $(<1.9 \mathrm{~mm})$ compared to the imaging resolution of the planning scans $\left(\mathrm{CT}=1.2 \times 1.2 \times 3 \mathrm{~mm}^{3}, \mathrm{MRI}=0.7 \times\right.$ $\left.0.7 \times 3.3 \mathrm{~mm}^{3}\right)$. Since EBRT doses were calculated with a 3 $\times 3 \times 3 \mathrm{~mm}^{3}$ resolution, dose points warped at this precision or lower were considered reliable. Moreover, since the EBRT dose is uniform near the high-dose volumes of brachytherapy, the impact of mismatches after DIR is limited. For bladder and rectum $\mathrm{D}_{2 \mathrm{~cm} 3}$, the difference between the values calculated with the UD and DIR method were indeed small. We therefore conclude that the DIR performance was sufficiently accurate for accumulating the bladder and rectum $\mathrm{D}_{2 \mathrm{~cm} 3}$.

Patients in this study were treated with smaller margins than for conventional VMAT/IMRT cervix plans to increase organ at risk sparing. Our results show that the EBRT dose near the BT boost can be considered uniform even when a conformal plan-of-the-day strategy is used. For patient treated at our facility with the currently available conformal treatment planning strategies, it is therefore not necessary to accumulate the bladder and rectum dose of EBRT and BT with DIR at the time of brachytherapy planning. Recently, planning strategies have been developed to create even more conformal EBRT dose distributions such as for proton therapy [30] or online MRI guidance [31]. It may be necessary to use DIR when accumulating the dose from EBRT and BT in these cases.

The small differences we found between the DIR and UD method are not unexpected, since for all patients a hotspot criterion $\left(\mathrm{D}_{1 \mathrm{~cm} 3}<103 \%\right.$ of the prescription dose) was used. When such a criterion is not used, the delivered EBRT dose near the planned BT boost may be non-uniform, and DIR may have added value over the UD method.

Four out of the ten patients were treated with an additional SIB boost to pathological lymph nodes. For these patients, the difference between DIR and UD was also small. Possibly, for patients receiving a SIB boost special care needs to be taken during EBRT planning to avoid a non-uniform dose near the planned BT high-dose volumes, such as the hotspot criterion used in this study.

In our previous study [8], differences between the DIR and UD method for the planned EBRT dose were investigated without considering the effect of interfraction motion between EBRT fractions and plan selection. Four patients from this previous study were also analyzed in this study. For the delivered EBRT dose calculated in our present study, differences between DIR and UD were up to 2.5 times larger than for the planned EBRT dose. Regardless whether the delivered or planned EBRT dose was investigated, the risk of late side effects remained within $5-10 \%$ for these patients.

A limitation of this study is the use of CBCT delineations to guide DIR. Due to the low contrast and imaging artifacts, CBCT images may be less suitable for delineations than CT or MRI. Both for bladder [32] and rectum
$[33,34]$, the inter-observer variation is small on CBCT, showing these structures can be recognized and delineated on CBCT.

In this study, the effects of intrafraction motion during EBRT were not incorporated in the estimation of the delivered EBRT dose. In a previous study [35], intrafraction motion during EBRT was studied for cervical cancer using bladder and rectum delineations of pre- and post-fraction CBCT scans. The change in rectum volume during one fraction was negligible but the average bladder volume changed by on average $62 \mathrm{~cm}^{3}$. However, in this study, the time interval between the pre-and post-fraction scan was $20.8 \mathrm{~min}$, while in our institute, the time interval is less than $7 \mathrm{~min}$. The dosimetric impact of intrafraction motion during EBRT is therefore limited for bladder and rectum.

\section{Conclusions}

For the accumulated $\mathrm{D}_{2 \mathrm{~cm} 3}$ in bladder and rectum from EBRT and BT, we found small differences between UD and DIR $(<4 \%)$. For the evaluation of $\mathrm{D}_{2 \mathrm{~cm} 3}$ it can be assumed that the VMAT/IMRT dose delivered with adaptive EBRT to bladder and rectum near the planned BT boost is uniform.

\section{Acknowledgements}

This work was funded by Elekta Brachytherapy (II 250008).

\section{Disclosure}

This work was funded by Elekta Brachytherapy. The department has a research collaboration with Elekta and Bel received grants from Elekta/Elekta Brachytherapy. Elekta Brachytherapy was not involved in the study design as well as in analysis, interpretation, and writing of the manuscript.

\section{References}

1. Bermudez A, Bhatla N, Leung E. Cancer of the cervix uteri. Int J Gynaecol Obstet 2015; 131 Suppl: S88-95.

2. Georg P, Pötter R, Georg D et al. Dose effect relationship for late side effects of the rectum and urinary bladder in magnetic resonance image-guided adaptive cervix cancer brachytherapy. Int J Radiat Oncol Biol Phys 2012; 82: 653-657.

3. Mazeron R, Fokdal LU, Kirchheiner KG et al. Dose-volume effect relationships for late rectal morbidity in patients treated with chemoradiation and MRI-guided adaptive brachytherapy for locally advanced cervical cancer: Results from the prospective multicenter EMBRACE study. Radiother Oncol 2016; 120: 412-419.

4. Pötter R, Haie-Meder C, Van Limbergen E et al. Recommendations from gynaecological (GYN) GEC ESTRO working group (II): concepts and terms in 3D image-based treatment planning in cervix cancer brachytherapy-3D dose volume parameters and aspects of 3D image-based anatomy, radiation physics, radiobiology. Radiother Oncol 2006; 78: 67-77.

5. Van de Schoot AJAJ, de Boer P, Visser J et al. Dosimetric advantages of a clinical daily adaptive plan selection strategy compared with a non-adaptive strategy in cervical cancer radiation therapy. Acta Oncol (Madr) 2017; 56: 667-674. 
6. Abe T, Tamaki T, Makino S et al. Assessing cumulative dose distributions in combined radiotherapy for cervical cancer using deformable image registration with pre-imaging preparations. Radiat Oncol 2014; 9: 293.

7. Hayashi K, Isohashi F, Akino Y et al. Estimation of the total rectal dose of radical external beam and intracavitary radiotherapy for uterine cervical cancer using the deformable image registration method. J Radiat Res 2015; 56: 546-552.

8. Van Heerden LE, Houweling AC, Koedooder C et al. Structure-based deformable image registration: Added value for dose accumulation of external beam radiotherapy and brachytherapy in cervical cancer. Radiother Oncol 2017; 123 319-324.

9. Kim H, Huq MS, Houser C et al. Mapping of dose distribution from IMRT onto MRI-guided high dose rate brachytherapy using deformable image registration for cervical cancer treatments: preliminary study with commercially available software. J Contemp Brachytherapy 2014; 6: 178-184.

10. Teo BK, Bonner Millar LP, Ding X, Lin LL. Assessment of cumulative external beam and intracavitary brachytherapy organ doses in gynecologic cancers using deformable dose summation. Radiother Oncol 2015; 115: 195-202.

11. Van de Schoot AJAJ, de Boer P, Visser J et al. Dosimetric advantages of a clinical daily adaptive plan selection strategy compared with a non-adaptive strategy in cervical cancer radiation therapy. Acta Oncol (Madr) 2017; 56: 667-674.

12. Vásquez Osorio EM, Hoogeman MS, Bondar L et al. A novel flexible framework with automatic feature correspondence optimization for nonrigid registration in radiotherapy. Med Phys 2009; 36: 2848.

13. Van Heerden LE, van Kesteren Z, Gurney-Champion OJ et al. Image distortions on a plastic interstitial CT/MR brachytherapy applicator at $3 \mathrm{~T}$ MRI and their dosimetric impact. Int J Radiat Oncol 2017; 99: 710-718.

14. Veiga C, McClelland J, Moinuddin S et al. Toward adaptive radiotherapy for head and neck patients: Feasibility study on using CT-to-CBCT deformable registration for 'dose of the day' calculations. Med Phys 2014; 41: 031703.

15. Stanley N, Glide-Hurst C, Kim J et al. Using patient-specific phantoms to evaluate deformable image registration algorithms for adaptive radiation therapy. J Appl Clin Med Phys 2013; 14: 4363.

16. Onozato Y, Kadoya N, Fujita Y et al. Evaluation of on-board $\mathrm{kV}$ cone beam computed tomography-based dose calculation with deformable image registration using hounsfield unit modifications. Int J Radiat Oncol 2014; 89: 416-423.

17. Kadoya N, Miyasaka Y, Yamamoto T et al. Evaluation of rectum and bladder dose accumulation from external beam radiotherapy and brachytherapy for cervical cancer using two different deformable image registration techniques. J Radiat Res 2017; 58: 920-728.

18. Hayashi $\mathrm{K}$, Isohashi $\mathrm{F}$, Akino $\mathrm{Y}$ et al. Estimation of the total rectal dose of radical external beam and intracavitary radiotherapy for uterine cervical cancer using the deformable image registration method. J Radiat Res 2015; 56: 546-552.

19. International Commission on Radiation Units and Measurements. Prescribing, Recording, and Reporting Brachytherapy for Cancer of the Cervix (ICRU report 89). Journal of the ICRU 2013; 13.

20. Pötter R, Haie-Meder C, Van Limbergen E et al. Recommendations from gynaecological (GYN) GEC ESTRO working group (II): concepts and terms in 3D image-based treatment planning in cervix cancer brachytherapy-3D dose volume parameters and aspects of 3D image-based anatomy, radiation physics, radiobiology. Radiother Oncol 2006; 78: 67-77.

21. Dice LR. Measures of the amount of ecologic association between species. Ecology 1945; 26: 297-302.
22. Bondar L, Hoogeman MS, Vásquez Osorio EM, Heijmen BJ. A symmetric nonrigid registration method to handle large organ deformations in cervical cancer patients. Med Phys 2010; 37: 3760.

23. Wognum S, Heethuis SE, Rosario T et al. Validation of deformable image registration algorithms on CT images of ex vivo porcine bladders with fiducial markers. Med Phys 2014; 41: 071916.

24. Kirisits C, Rivard MJ, Baltas D, Ballester F et al. Review of clinical brachytherapy uncertainties: analysis guidelines of GEC-ESTRO and the AAPM. Radiother Oncol 2014; 110: 199-212.

25. Nesvacil N, Tanderup K, Lindegaard JC et al. Can reduction of uncertainties in cervix cancer brachytherapy potentially improve clinical outcome? Radiother Oncol 2016; 120: 390-396.

26. Hellebust TP, Tanderup K, Lervåg C et al. Dosimetric impact of interobserver variability in MRI-based delineation for cervical cancer brachytherapy. Radiother Oncol 2013; 107: 13-19.

27. Nesvacil N, Tanderup K, Hellebust TP et al. A multicentre comparison of the dosimetric impact of inter- and intra-fractional anatomical variations in fractionated cervix cancer brachytherapy. Radiother Oncol 2013; 107: 20-25.

28. Georg P, Pötter R, Georg D et al. Dose effect relationship for late side effects of the rectum and urinary bladder in magnetic resonance image-guided adaptive cervix cancer brachytherapy. Int J Radiat Oncol Biol Phys 2012; 82: 653-657.

29. Mazeron R, Fokdal LU, Kirchheiner K et al. Dose-volume effect relationships for late rectal morbidity in patients treated with chemoradiation and MRI-guided adaptive brachytherapy for locally advanced cervical cancer: Results from the prospective multicenter EMBRACE study. Radiother Oncol 2016; 120: 412-419.

30. Van de Schoot AJAJ, de Boer P et al. Dosimetric advantages of proton therapy compared with photon therapy using an adaptive strategy in cervical cancer. Acta Oncol (Madr) 2016; 55: 892-899.

31. Kerkhof EM, Raaymakers BW, van der Heide UA et al. Online MRI guidance for healthy tissue sparing in patients with cervical cancer: An IMRT planning study. Radiother Oncol 2008; 88: 241-249.

32. Nishioka K, Shimizu S, Kinoshita R et al. Evaluation of inter-observer variability of bladder boundary delineation on cone-beam CT. Radiat Oncol 2013; 8: 185.

33. Foroudi F, Haworth A, Pangehel A et al. Inter-observer variability of clinical target volume delineation for bladder cancer using CT and cone beam CT. J Med Imaging Radiat Oncol 2009; 53: 100-106

34. Lütgendorf-Caucig C, Fotina I, Stock M et al. Feasibility of CBCT-based target and normal structure delineation in prostate cancer radiotherapy: Multi-observer and image multi-modality study. Radiother Oncol 2011; 98: 154-161.

35. Heijkoop ST, Langerak TR, Quint S et al. Quantification of intra-fraction changes during radiotherapy of cervical cancer assessed with pre- and post-fraction Cone Beam CT scans. Radiother Oncol 2015; 117: 536-541. 